\title{
EPIDEMIOLOGY
}

\section{Revisiting family history}

l: $\mathrm{t}$ is well known that a family history of cancer is a key factor in someone's risk of developing this disease and, therefore, individuals with a family history of cancer are encouraged to begin screening earlier and with moresensitive methods than individuals at average risk. That is one of the reasons why the Agency of Healthcare Research and Quality recommends primary care clinicians to compile a detailed family history that includes whether, at what age and what type of cancer close relatives were diagnosed with. This is especially recommended in cases of colorectal, prostate and breast cancer, as these types of cancer are particularly affected by family history. For example, having one firstdegree relative (mother, sister, or daughter) with breast cancer approximately doubles the risk that a woman will develop this type of cancer, and having two firstdegree relatives increases this risk by up to threefold. But, how does cancer family history change with time? How often do changes occur that would modify the recommended screening? Dianne Finkelstein and her collaborators have now provided some answers to these questions.

\section{But, how does cancer family history change with time? How often do changes occur that would modify the recommended screening? 77}

The researchers were interested in finding out how often changes in a person's family history of cancer over time would affect the cancer screening schedule and tests recommended by standard guidelines. To address this question, they used two complementary analytical approaches-a retrospective and a prospective analysis - with different sets of data of family history of colorectal, breast and prostate cancer, obtained from the Cancer Genetics Network (CGN). The CGN is a registry with detailed

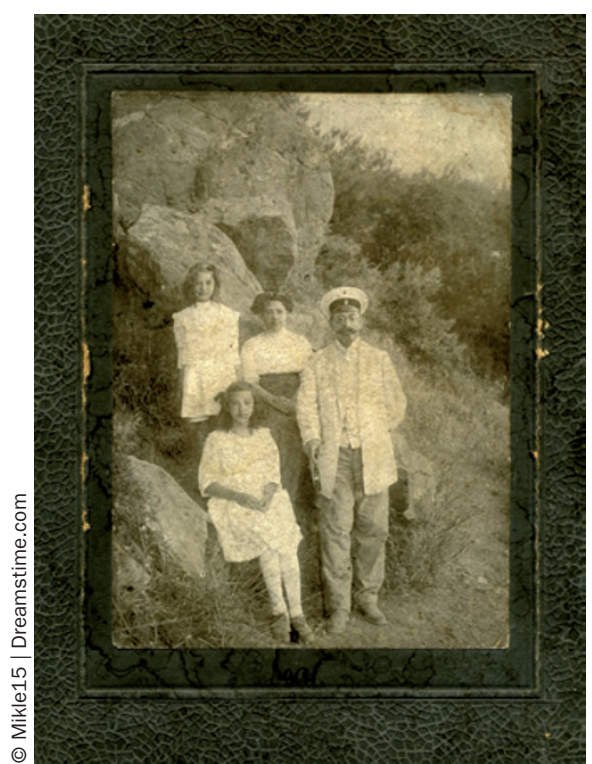

information of individuals with a personal or family history of cancer that serves as a resource for investigators wishing to study the inherited predisposition to this disease.

"For the retrospective analysis, we constructed a data set for each participant with an observation corresponding to each year of life up until entry into the CGN, and for each year we evaluated whether they had clinically relevant family history," explains Finkelstein. This retrospective study included more than 11,000 individuals, from whom follow-up data on 1,900 were used in a prospective study. "For the prospective analysis, we determined the number of participants who, at CGN enrollment, did not meet the criteria for high-risk screening," says Finkelstein. The information of these participants was updated on an annual basis for an average of 8 years. The researchers then determined how many participants would have been classified as meeting the high-risk criteria according to the updated information about their cancer family history. Then they evaluated the 10-year rate at which the cancer family history of these participants changes so that they would meet criteria for needing earlier or more-intensive screening.
Indeed, the results of both studies revealed that family history changes notably and, between the ages of 30 and 50 years, the percentage of individuals at high risk of developing breast and colorectal cancer increased considerably. As Sharon Plon, senior author of the study, details, "for colorectal cancer, at age 30 , only $2 \%$ were in a higher risk group but by age $50,7 \%$ of individuals were considered high risk. Thus, between ages 30 and 50, an additional $5 \%$ of people reported a significant enough change in their family history to justify starting colonoscopy screening before age 50 , the age when average-risk individuals should be getting colonoscopies". The results were similar for breast cancer, as between the ages of 30 and 50 years, $4 \%$ of women in the study had changes in their breast cancer family history that would prompt their doctor to recommend getting an MRI scan in addition to their annual mammogram. Results from the prostate cancer analyses, however, were less conclusive.

The researchers hope that this study will encourage patients to be aware of details of any close relatives who might have been diagnosed with cancer, as this is an important aspect of their own health care. This should also prompt clinicians to take comprehensive family histories of new patients and update those histories every 5 to 10 years, especially for patients between the ages of 30 and 50 years. But, will updating family history information help in detecting early colorectal or breast cancer? "It would be useful to do a controlled study, using the best educational approach and schedule of updates to determine whether this policy would increase the number of early-stage cancers that are detected, and ultimately impact the cancer death rate", concludes Finkelstein.

\section{Teresa Villanueva}

Original article Ziogas, A. et al. Clinically relevant changes in family history of cancer over time. JAMA 306, 172-178 (2011) 